\title{
Qualifikationen von Lehrenden in der beruflich-betrieblichen Weiterbildung
}

\author{
Susanne Wißhak $(\mathbb{D})$ Caroline Bonnes $(\mathbb{D}) \cdot$ Inka Keller • Dorothee Barth • \\ Sabine Hochholdinger $(\mathbb{D}$
}

Angenommen: 13. Januar 2020 / Online publiziert: 24. Januar 2020

(C) Der/die Autor(en) 2020

Zusammenfassung Über die Qualifikationen von Lehrenden in der beruflich-betrieblichen Weiterbildung in Deutschland ist bisher wenig bekannt, da sie in den meisten Erhebungen zum Weiterbildungspersonal unterrepräsentiert und/oder als Teilgruppe nicht explizit ausgewiesen sind. Der vorliegende Beitrag untersucht daher deskriptiv die Qualifikationen von $N=896$ Lehrenden in der beruflich-betrieblichen Weiterbildung und vergleicht die Befunde mit den Ergebnissen anderer Studien. Die Lehrenden gaben ähnlich häufig an, einen Hochschulabschluss zu besitzen (70,9\%), allerdings wurden pädagogische Hochschulabschlüsse seltener genannt $(16,5 \%)$. Trainerausbildungen und/oder andere Zusatzqualifikationen wurden dagegen häufiger angegeben $(86,4 \%)$. Insgesamt scheinen Lehrende in der beruflichbetrieblichen Weiterbildung ein hohes Qualifikationsniveau zu besitzen, wobei es sich jedoch selten um ausgebildete Pädagog/innen handelt. Im Hinblick auf die Professionalisierung im Weiterbildungsbereich erscheint es lohnend, diese Teilgruppe des Weiterbildungspersonals als solche zukünftig genauer zu untersuchen.

Schlüsselwörter Qualifikationen · Lehrende $\cdot$ Beruflich-betriebliche Weiterbildung $\cdot$ Professionalisierung $\cdot$ Weiterbildungspersonal $\cdot$ Trainer

\section{Qualifications of trainers in continuing vocational training}

\author{
Abstract Little is known so far about the qualifications of trainers in continuing \\ vocational training in Germany, as they are underrepresented in most surveys re- \\ garding adult education personnel and/or are not explicitly identified as a subgroup. \\ S. Wißhak $(\bowtie) \cdot$ C. Bonnes $\cdot$ D. Barth $\cdot$ S. Hochholdinger \\ Lehrstuhl Betriebspädagogik, Fach125, Universität Konstanz, 78457 Konstanz, Deutschland \\ E-Mail: Susanne.Wisshak@uni-konstanz.de \\ I. Keller \\ Arbeitsstelle Hochschuldidaktik, Fach 227 I 206, Universität Konstanz, 78457 Konstanz, Deutschland
}


This article therefore describes the qualifications of $N=896$ trainers in continuing vocational training and compares the findings to the results of other studies. Trainers similarly frequently stated that they had a university degree (70.9\%), but pedagogical university degrees were mentioned less frequently (16.5\%). Train-the-trainer certificates and/or other additional qualifications, on the other hand, were mentioned more frequently (86.4\%). Overall, trainers in continuing vocational training appear to have a high level of qualification, although they rarely are trained pedagogues. With regard to professionalization in the continuing education sector, it seems worthwhile to examine this subgroup of adult education personnel in more detail in the future.

Keywords Qualifications · Trainers · Continuing vocational training · Professionalization · Adult education personnel

\section{Begründung des Forschungsinteresses und Fragestellungen}

Die Ausbildung des Lehrpersonals als zentrale Determinante der Unterrichtsqualität rückt in der Bildungsforschung zunehmend in den Fokus. So hat sich in der empirischen Unterrichtsforschung eine breite Forschungslandschaft zur Ausbildung und zum Kompetenzerwerb von Lehrkräften etabliert (z.B. Kunina-Habenicht et al. 2013; Kunter et al. 2013). Es wird von einer Wirkungskette ausgegangen, der zufolge einschlägige Lerngelegenheiten in den verschiedenen Ausbildungsphasen den Erwerb professioneller Handlungskompetenz der Lehrenden beeinflussen, während diese Kompetenz wiederum einen positiven Zusammenhang mit der Lehrqualität und den Lernerfolgen aufweist (Diez 2010; Marx et al. 2018).

Auch in der Weiterbildungsforschung wird zunehmend versucht, die heterogenen Qualifikationen des Lehrpersonals systematisch zu erfassen. Die größten Erhebungen zum Weiterbildungspersonal in Deutschland sind die WSF-Studie (2005), die Studie Beschäftigung in der Weiterbildung (Alfänger et al. 2016) und der wb-personalmonitor (Autorengruppe wb-personalmonitor 2016). In den Studien wird teilweise zwischen allgemeiner und beruflicher Weiterbildung unterschieden. Lehrende in der beruflich-betrieblichen Weiterbildung, die sich selbst häufig als Trainer/innen bezeichnen, stehen jedoch nicht im Fokus. Die beruflich-betriebliche Weiterbildung wird hier in Abgrenzung zur allgemeinen Weiterbildung verstanden. Dazu gehört die individuelle berufsbezogene Weiterbildung sowie vor allem auch die betriebliche Weiterbildung, die den weitaus größeren Anteil der Weiterbildungsaktivitäten ausmacht (Bilger und Strauß 2017). Zur betrieblichen Weiterbildung gehören unternehmensinterne, aber auch in Auftrag gegebene Weiterbildungsmaßnahmen. Die Lehrenden in der beruflich-betrieblichen Weiterbildung sind meist hauptberuflich tätig (Fuchs 2011). Sie sind außerdem meist selbständig, bzw. bieten als externe Lehrkräfte auf freiberuflicher Basis Trainings für verschiedene Betriebe bzw. Unternehmen an (Fuchs 2011; Wißhak und Hochholdinger 2019).

$\mathrm{Da}$ in den bisherigen Untersuchungen zum Weiterbildungspersonal der Zugang zu den Befragten meist über Weiterbildungsträger stattfand, blieben diejenigen Trainer/innen, die auf selbständiger Basis für Unternehmen tätig sind, ohne dabei für 
eine Weiterbildungsorganisation zu arbeiten, unberücksichtigt (WSF 2005). Darüber hinaus wurde die innerbetriebliche Weiterbildung, die von Unternehmen nur für ihre eigenen Mitarbeitenden angeboten wird, in der WSF Studie (2005) und dem wbpersonalmonitor (Koscheck und Martin 2016) nicht erfasst, sowie in der Studie von Alfänger et al. (2016) nur in sehr geringem Umfang.

Die betriebliche Weiterbildung macht jedoch einen Großteil der Weiterbildung in Deutschland aus. So umfasste sie im Jahr 2016 mehr als die Hälfte (71\%) aller Weiterbildungsaktivitäten und $49 \%$ des Zeitvolumens der Weiterbildung (Bilger und Strauß 2017). Die geringe Berücksichtigung des betrieblichen Lehrpersonals in den genannten Studien bei gleichzeitig hohem Anteil betrieblicher Weiterbildungsmaßnahmen in Deutschland lässt darauf schließen, dass die bisherige Datenlage noch kein vollständiges Bild der Qualifikationen von Lehrenden in der Weiterbildung abgibt.

Studien, die sich explizit mit den Qualifikationen bzw. Berufsbiografien von Trainer/innen in Deutschland befassen, gibt es nur vereinzelt (Bonnes und Hochholdinger 2016; Fuchs 2011). Auch in Arbeitsmarktstatistiken kommen Lehrende in der beruflich-betrieblichen Weiterbildung mangels einer definierten Berufsklassifikation nicht vor (Käpplinger und Lichte 2012). Die mangelhafte Datenlage ist auch darauf zurückzuführen, dass sie weder vollständig durch einen Berufsverband vertreten, noch in einer anderen Form gemeinsam organisiert und erreichbar sind. Somit ist über die Qualifikationen der Lehrenden in der beruflich-betrieblichen Weiterbildung wenig bekannt. Dies wäre jedoch wichtig, um ihren Kompetenzerwerb und ihre Professionalisierung in Zukunft besser analysieren und fördern zu können. Dabei ist zunächst keine Etablierung einer kollektiven Profession gemeint, die in der Erwachsenenbildung/Weiterbildung derzeit unrealistisch scheint, sondern die individuelle Professionalität im Sinne der Befähigung zu professionellem Handeln der Lehrpersonen (Gieseke 2018).

Hinzu kommt, dass Befunde von Marx et al. (2018) darauf hinweisen, dass Lehrende in der beruflich-betrieblichen Weiterbildung im Vergleich zu Lehrenden in der allgemeinen Weiterbildung unterschiedliche Wissensstände aufweisen. In der Studie mit $N=209$ Lehrenden besaßen die Trainer/innen aus dem beruflich-betrieblichen Bereich signifikant mehr Wissen über Lehr-Lernmethoden und -konzepte, als die Lehrenden aus der allgemeinen Weiterbildung. Die Autor/innen vermuten an dieser Stelle Unterschiede in der Entwicklung des professionellen Handlungswissens, die mit den beruflichen Gegebenheiten der Lehrenden zusammenhängen könnten. Trainer/innen in der beruflich-betrieblichen Weiterbildung begegnen aufgrund des professionellen Kontexts möglicherweise einer höheren Anspruchshaltung und müssen, da sie ihre Tätigkeit meist hauptberuflich und selbständig verüben, diesen Ansprüchen genügen, um ihren Lebensunterhalt zu sichern. Marx et al. fordern deshalb, den Weiterbildungsbereich, in dem die Lehrenden tätig sind, in zukünftigen Untersuchungen zu berücksichtigen.

Es lässt sich also zum einen eine Forschungslücke bezüglich der Qualifikationen von Lehrenden in der beruflich-betrieblichen Weiterbildung feststellen. Zum anderen stellt sich die Frage, ob sich, wenn man diese untersucht, Unterschiede zu den Befunden der übrigen Studien ergeben. 
Der Untersuchung liegen daher folgende Fragen zugrunde:

1. Über welche Qualifikationen verfügen Lehrende in der beruflich-betrieblichen Weiterbildung?

2. Gibt es Unterschiede bezüglich Art und Umfang der verschiedenen Qualifikationen im Vergleich zu Befunden bisheriger Studien zum Weiterbildungspersonal?

\section{Forschungslage und Annahmen}

Im Folgenden wird der aktuelle Stand der Forschung vorgestellt. In Abschn. 2.1 wird zunächst beschrieben, welche Personengruppen in den bisherigen Studien untersucht wurden. Neben der Stichprobengröße werden Informationen darüber berichtet, in welchem Land oder Bundesland die Erhebung stattfand, ob die Befragten in der allgemeinen, in der beruflichen, oder in der beruflich-betrieblichen Weiterbildung tätig waren, und ob es sich um Lehrpersonen oder anderweitig in der Weiterbildung Tätige handelt. In Abschn. 2.2 werden die jeweiligen Befunde zu den Qualifikationen der Befragten vorgestellt. Es folgen die Annahmen der vorliegenden Studie in Abschn. 2.3.

\subsection{Erhebungen zum Weiterbildungspersonal in Deutschland}

Das Institut Wirtschafts- und Sozialforschung (WSF 2005) befragte im Auftrag des Bundesministeriums für Bildung und Forschung (BMBF) Lehrende, die bei Weiterbildungsanbietern der politischen, beruflichen und allgemeinen Weiterbildung in Deutschland beschäftigt waren. Aufgrund der verbreiteten Mehrfachbeschäftigung von Lehrenden in der Weiterbildung spiegeln die 4847 ausgewerteten Fragebögen Beschäftigungsverhältnisse wider und nicht Personen. Von den befragten Trägern gaben $8,1 \%$ an, in der allgemeinen und politischen Weiterbildung tätig zu sein, $67,0 \%$ gaben berufliche Weiterbildung an und 24,9\% gaben an, sowohl in der beruflichen als auch in der allgemeinen und politischen Weiterbildung aktiv zu sein. Bei $83 \%$ der Tätigkeitsverhältnisse wurde angegeben, ausschließlich oder überwiegend lehrend tätig zu sein.

Lenk (2010) befragte 501 freiberuflich in der Weiterbildung Tätige in Hessen und darüber hinaus. Bei der Frage nach ihren Kernaktivitäten mit mehrfacher Antwortmöglichkeit gaben $86 \%$ der Befragten die Kategorie Unterrichten/Lehren/Leiten an. Es ist unklar, inwiefern die Befragten in der allgemeinen oder in der beruflichbetrieblichen Weiterbildung tätig sind.

Die Studie von Fuchs (2011) ist die einzige, in der ausdrücklich Trainer/innen $(N=323)$ befragt wurden. Hiermit sind Lehrende in der beruflichen oder betrieblichen Weiterbildung gemeint. Aufgrund der offenen Online-Befragung ist nicht auszuschließen, dass einige auch außerhalb Deutschlands tätig waren.

Schütz und Nittel (2012) werteten die Qualifikationen von 144 im Berufsfeld Erwachsenenbildung tätigen Personen aus, die aus einer größeren Untersuchung mit 1601 pädagogisch tätigen Personen stammen. Ob die Erwachsenenbildner/innen der 
beruflichen oder allgemeinen Weiterbildung zuzuordnen sind, und zu welchem Grad sie lehrend tätig sind, geht nicht aus der Quelle hervor.

Der Mikrozensus ist eine vom Statistischen Bundesamt durchgeführte bundesweite Erhebung, bei der anhand von Klumpenstichproben deutsche Haushalte befragt werden. In die Auswertung zum Weiterbildungspersonal von Martin und Langemeyer (2014) wurden 1854 Personen einbezogen, die in ihrer ersten Erwerbstätigkeit ein Einkommen in der Erwachsenenbildungsbranche erzielten. Hier wurden die Befragten also nicht über Weiterbildungsorganisationen gewonnen, sodass auch freiberufliche Weiterbildner/innen erfasst wurden, die bspw. direkt für Unternehmen arbeiten. Der Anteil der Lehrenden in dieser Personengruppe wird jedoch nicht ausgewiesen.

In der Studie von Alfänger et al. (2016) wurden über eine offene Online-Erhebung die Daten zu 1958 in der Weiterbildung Tätigen ausgewertet. Ungefähr die Hälfte der Teilnehmenden $(51,9 \%)$ ist in der öffentlich finanzierten beruflichen Weiterbildung tätig, 40,9\% in der öffentlich finanzierten allgemeinen Weiterbildung und nur 7,2\% in der privat oder betrieblich finanzierten Weiterbildung. Die Stichprobe umfasst sowohl lehrendes als auch planendes Personal, wobei 54\% der Personen angaben, u. a. oder überwiegend in der Lehre tätig zu sein. Da es sich um eine offene OnlineErhebung handelt, ist nicht auszuschließen, dass auch Weiterbildner/innen außerhalb Deutschlands den Fragebogen ausgefüllt haben.

Der Zugang zu den 6062 im Rahmen des wb-personalmonitors befragten Weiterbildner/innen erfolgte über deutsche Weiterbildungseinrichtungen, die Weiterbildung offen zugänglich anbieten. Die Befragten waren bei folgenden Einrichtungstypen beschäftigt: Privat-kommerziell $(20,1 \%)$, privat-gemeinnützig $(11,5 \%)$, betrieblich oder wirtschaftsnah (14,2\%), berufliche Schulen oder (Fach-)Hochschulen $(11,5 \%)$, Volkshochschulen $(27 \%)$ und Einrichtungen von Kirchen, Parteien, etc. $(12,6 \%)$ (Koscheck und Martin 2016). Die Studie schloss alle Tätigkeitsfelder in den Einrichtungen ein, also die Leitung, Planung, Verwaltung, Lehre und Beratung (Martin et al. 2016). Dabei stellen die Lehrenden die größte Subgruppe dar. Eine reine Lehrtätigkeit wurde bei $28,7 \%$ der Tätigkeitsverhältnisse angegeben (Martin 2016). Der überwiegende Anteil der Tätigkeitsverhältnisse $(58,0 \%)$ umfasst Lehre und andere Tätigkeiten. Lediglich 13,3\% der Tätigkeitsverhältnisse enthalten keine Lehrtätigkeit.

Marx et al. (2018) untersuchten 209 Lehrkräfte in Deutschland, von denen knapp die Hälfte $(46,3 \%)$ in der allgemeinen Weiterbildung und die andere Hälfte in der beruflich-betrieblichen Weiterbildung tätig waren. Der Zugang zu den Lehrenden erfolgte für den allgemeinen Weiterbildungsbereich über Volkshochschulen in verschiedenen Bundesländern und für den beruflich-betrieblichen Bereich über deutsche Trainernetzwerke.

Zusammenfassend wird deutlich, dass die bisherigen Stichproben nur zum Teil Lehrende in der beruflichen Weiterbildung beinhalten und $\mathrm{zu}$ einem noch kleineren Anteil Lehrende in der beruflich-betrieblichen Weiterbildung berücksichtigen (Tab. 1). Sowohl in der Studie des WSF (2005) als auch von Alfänger et al. (2016) liegt der Schwerpunkt auf der öffentlichen beruflichen Weiterbildung. Die Stichprobe von Marx et al. (2018) weist ca. zur Hälfte Lehrende aus der beruflich-betrieblichen Weiterbildung auf. In den übrigen Studien (Lenk 2010; Schütz und Nittel 2012; Martin und Langemeyer 2014; Autorengruppe wb-personalmonitor 2016) gibt es 
Tab. 1 Übersicht über den Anteil beruflicher bzw. beruflich-betrieblicher Weiterbildung und den Anteil der lehrenden Personen in den bisherigen Studien

\begin{tabular}{|c|c|c|c|}
\hline Quelle & $N$ & $\begin{array}{l}\text { Anteil beruflicher bzw. beruflich-be- } \\
\text { trieblicher Weiterbildung }\end{array}$ & $\begin{array}{l}\text { Anteil der Lehren- } \\
\text { den Personen }\end{array}$ \\
\hline Fuchs (2011) & 323 & $\begin{array}{l}100 \% \text { in der beruflich-betrieblichen } \\
\text { Weiterbildung Tätige }\end{array}$ & $\begin{array}{l}100 \% \text { der Befrag- } \\
\text { ten sind in der } \\
\text { Lehre tätig }\end{array}$ \\
\hline $\begin{array}{l}\text { Marx et al. } \\
(2018)\end{array}$ & 209 & $\begin{array}{l}53,7 \% \text { in der beruflich-betrieblichen } \\
\text { Weiterbildung Tätige }\end{array}$ & $\begin{array}{l}100 \% \text { der Befrag- } \\
\text { ten sind in der } \\
\text { Lehre tätig }\end{array}$ \\
\hline $\begin{array}{l}\text { Alfänger et al. } \\
(2016)\end{array}$ & 1958 & $\begin{array}{l}51,9 \% \text { in der öffentlich finanzierten } \\
\text { beruflichen Weiterbildung, } 7,2 \% \text { in } \\
\text { der privat oder betrieblich finanzierten } \\
\text { Weiterbildung Tätige }\end{array}$ & $\begin{array}{l}54 \% \text { der Befragten } \\
\text { sind (u. a.) in der } \\
\text { Lehre tätig }\end{array}$ \\
\hline WSF (2005) & $\begin{array}{l}5109 \text { Weiter- } \\
\text { bildungsträger } \\
4847 \text { Beschäf- } \\
\text { tigte }\end{array}$ & $\begin{array}{l}\text { 91,9\% der Träger gaben an, u. a. in } \\
\text { der beruflichen Weiterbildung aktiv zu } \\
\text { sein. Die betriebliche Weiterbildung } \\
\text { wurde nicht erfasst }\end{array}$ & $\begin{array}{l}83 \% \text { der Beschäf- } \\
\text { tigungsverhältnisse } \\
\text { sind (u. a.) in der } \\
\text { Lehre }\end{array}$ \\
\hline $\begin{array}{l}\text { Autorengruppe } \\
\text { wb-personal- } \\
\text { monitor (2016), } \\
\text { Martin (2016) }\end{array}$ & 6062 & k. A. & $\begin{array}{l}86,7 \% \text { der Befrag- } \\
\text { ten sind (u. a.) in } \\
\text { der Lehre tätig }\end{array}$ \\
\hline Lenk (2010) & 501 & k. A. & $\begin{array}{l}86 \% \text { der Befragten } \\
\text { sind (u. a.) in der } \\
\text { Lehre tätig }\end{array}$ \\
\hline $\begin{array}{l}\text { Schütz und Nittel } \\
\text { (2012) }\end{array}$ & 144 & k. A. & Keine Angabe \\
\hline $\begin{array}{l}\text { Martin und Lan- } \\
\text { gemeyer (2014) }\end{array}$ & 1854 & k. A. & Keine Angabe \\
\hline
\end{tabular}

keine genaueren Angaben, ob es sich um Personen aus der allgemeinen, oder aus der beruflichen bzw. beruflich-betrieblichen Weiterbildung handelt. Lediglich die Erhebung von Fuchs (2011) legt den Schwerpunkt auf Trainer/innen in der beruflichbetrieblichen Weiterbildung.

In Bezug auf die Lehrtätigkeit untersuchen die meisten der Studien Personen, die vorwiegend oder zumindest zu einem Teil Lehrtätigkeiten ausüben (WSF 2005; Lenk 2010; Fuchs 2011; Autorengruppe wb-personalmonitor 2016; Marx et al. 2018). In der Studie von Alfänger et al. (2016) üben nur ca. die Hälfte eine Lehrtätigkeit aus. Für Schütz und Nittel (2012) sowie Martin und Langemeyer (2014) fehlen diese Angaben.

\subsection{Befunde zu Qualifikationen von Beschäftigten in der Weiterbildung}

Entsprechend der großen institutionellen Heterogenität des Weiterbildungssektors in Deutschland zeichnen sich die in diesem Bereich Tätigen durch eine große Vielfalt ihrer Bildungs- und Berufsbiografien aus (Autorengruppe wb-personalmonitor 2016; Dobischat et al. 2018). Im Folgenden wird ein Überblick über bisherige Befunde zu Qualifikationen des Weiterbildungspersonals in Deutschland gegeben (Tab. 2). 


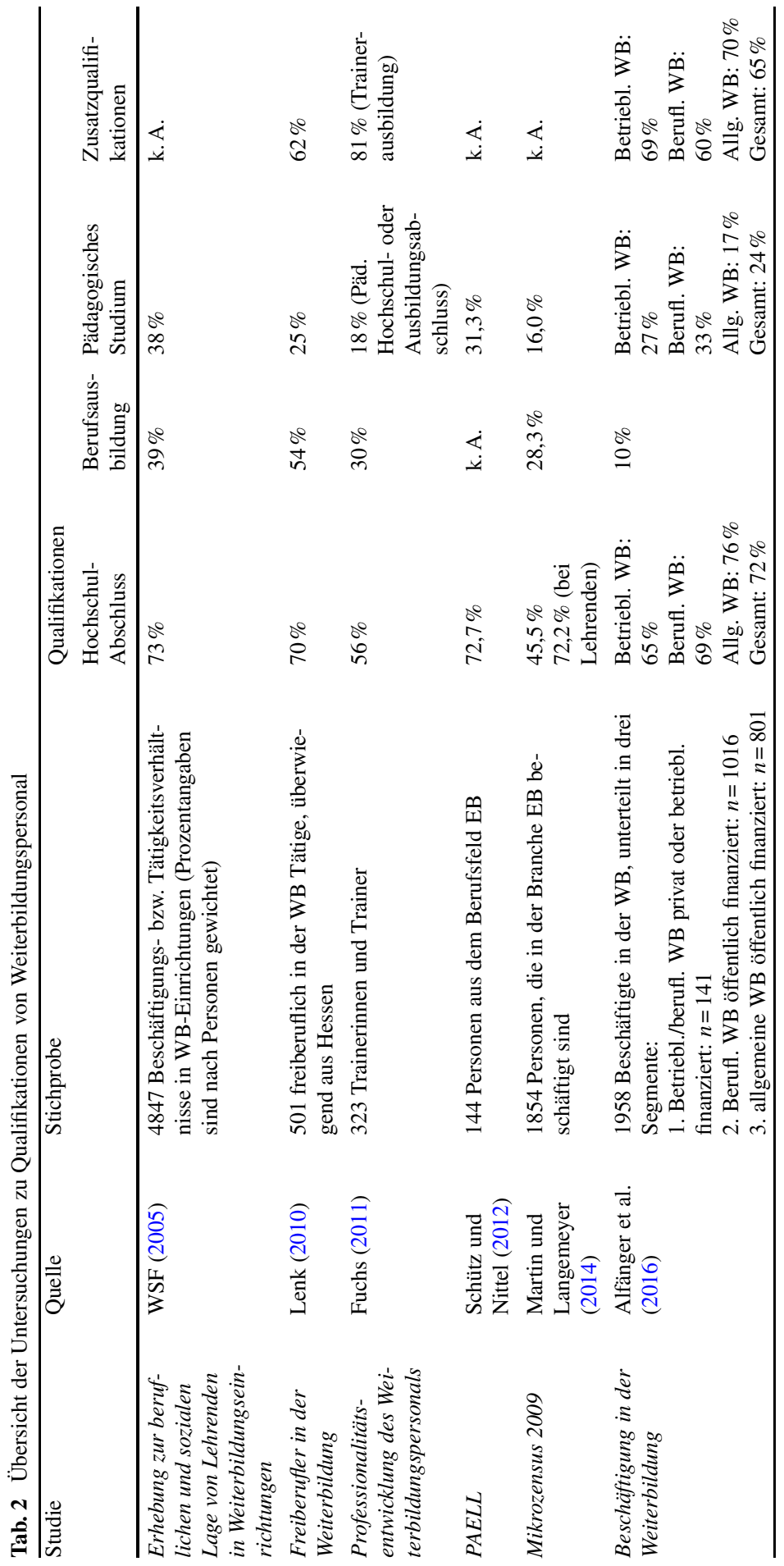




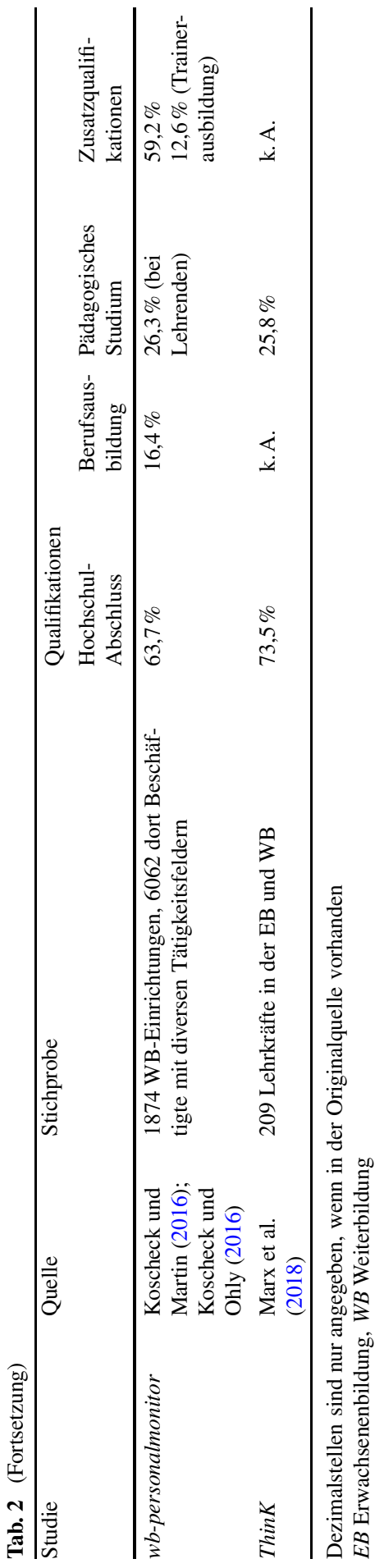


Für das Weiterbildungspersonal gibt es in Deutschland keine obligatorische Ausbildung. Das Studium der Erwachsenen- bzw. Weiterbildung soll für leitende bzw. disponierende Tätigkeiten in Weiterbildungseinrichtungen qualifizieren und bietet wenig Inhalte, die auf eine Lehrtätigkeit vorbereiten (Wißhak und Hochholdinger 2016). Die Lehrenden gelangen häufig über ihre fachliche Qualifikation wie bspw. Sprachen, IT-Kenntnisse oder Erfahrung im Vertrieb zu ihrer Tätigkeit in der Weiterbildung (Bonnes und Hochholdinger; Fuchs 2011; Koscheck und Ohly 2016). Viele Lehrpersonen besitzen Zusatzqualifikationen wie Train-the-Trainer-Zertifikate oder Ausbildungen in Beratung und Coaching (Koscheck und Ohly 2016). Trotz der heterogenen Qualifikationen von Weiterbildungspersonal soll in diesem Kapitel versucht werden, die wichtigsten Befunde zusammenzufassen.

\subsubsection{Hochschul- und Berufsabschlüsse}

Aus bisherigen Untersuchungen zum Weiterbildungspersonal geht hervor, dass dieses überwiegend hohe Bildungsabschlüsse aufweist. Im wb-personalmonitor gaben 63,7\% der Befragten an, einen Hochschulabschluss zu besitzen (Koscheck und Ohly 2016). Lediglich 16,4\% gaben als höchste berufliche Qualifikation eine Berufsausbildung und 17,0\% eine Fachausbildung an. Bei den übrigen in Tab. 2 zusammengefassten Studien lag der Akademikeranteil zwischen 45,5\% bei der Auswertung des Mikrozensus durch Martin und Langemeyer (2014) und 73,5\% in der ThinK Studie von Marx et al. (2018).

In den meisten Studien wird außerdem der Anteil pädagogischer Hochschulabschlüsse berichtet, da davon ausgegangen wird, dass diese trotz der Heterogenität im Weiterbildungsbereich als Qualifikationsnachweis gelten. Der Anteil liegt zwischen $18 \%$ pädagogischer Berufs- und Hochschulabschlüsse bei Fuchs (2011) und $38 \%$ pädagogischer Hochschulabschlüsse in der WSF-Studie (2005).

\subsubsection{Zusatzqualifikationen}

Im wb-personalmonitor gaben 59,2\% des Weiterbildungspersonals an, mindestens eine Zusatzqualifikation zu besitzen. Dabei wurde die Ausbildereignung nach der Ausbildungseignungsverordnung (AEVO) am häufigsten genannt (23,3\% der Lehrenden), gefolgt von Coaching- und Trainerausbildungen (12,9 und 12,6\% der Lehrenden). Die Vermutung von Koscheck und Ohly (2016), dass Lehrende ohne pädagogischen Hochschulabschluss in größerem Umfang Zusatzqualifikationen erwerben, bestätigte sich nicht. Dafür zeigte sich, dass Absolventinnen und Absolventen pädagogischer Studiengänge häufiger Ausbildungen im Bereich Beratung und Coaching besaßen. Lenk (2010) berichtet $62 \%$ der freiberuflich in der Weiterbildung Tätigen mit Zusatzqualifikationen, bei Alfänger et al. waren es 65\%. Fuchs (2011) fragte gezielt nach Trainerausbildungen und berichtet $81 \%$. 


\subsection{Annahmen zu den Qualifikationen von Lehrenden in der beruflich- betrieblichen Weiterbildung}

Bei den Lehrenden in der beruflich-betrieblichen Weiterbildung handelt es sich um eine Untergruppe des Weiterbildungspersonals, das in den oben aufgeführten Studien untersucht wurde. Daher gehen wir zunächst davon aus, dass sie sich bezüglich ihrer berufsbildenden Abschlüsse und Zusatzqualifikationen nicht maßgeblich vom übrigen Weiterbildungspersonal unterscheiden, sofern in bisherigen Studien kein abweichender Befund vorliegt. Daraus ergeben sich folgende, zu prüfende Annahmen:

\subsubsection{Hochschul- und Berufsabschlüsse}

Annahme 1a. Lehrende in der beruflich-betrieblichen Weiterbildung besitzen ähnlich häufig Hochschulabschlüsse wie das bisher untersuchte Weiterbildungspersonal.

Annahme 1b. Lehrende in der beruflich-betrieblichen Weiterbildung besitzen ähnlich häufig einen pädagogischen Hochschulabschluss wie das bisher untersuchte Weiterbildungspersonal.

Annahme 1c. Lehrende in der beruflich-betrieblichen Weiterbildung besitzen ähnlich häufig Berufsausbildungen wie das bisher untersuchte Weiterbildungspersonal.

\subsubsection{Zusatzqualifikationen}

Annahme 2a. Lehrende in der beruflich-betrieblichen Weiterbildung besitzen ebenso häufig mindestens eine Zusatzqualifikation wie das bisher untersuchte Weiterbildungspersonal.

Annahme 2b. Aufgrund der Studie von Fuchs (2011) zu Trainer/innen wird angenommen, dass Lehrende in der beruflich-betrieblichen Weiterbildung häufiger eine Trainerausbildung bzw. ein Train-the-Trainer Zertifikat besitzen als das bisher untersuchte Weiterbildungspersonal.

\subsubsection{Pädagogische Hochschulabschlüsse und Zusatzqualifikationen}

Annahme 3a. Für unsere Stichprobe wird angenommen, dass sich Lehrende mit und ohne pädagogischen Hochschulabschluss nicht bezüglich der Häufigkeit unterscheiden, mit der sie mindestens eine Zusatzqualifikation besitzen.

Annahme 3b. Wir erwarten keinen Unterschied bezüglich der Häufigkeit, mit der die Befragten in unserer Stichprobe mit und ohne pädagogischen Hochschulabschluss eine Trainerausbildung absolviert haben.

Die Annahmen 3a und 3b werden anhand eines Chi-Quadrat-Tests überprüft. 
Tab. 3 Übersicht Studien $(N=896)$

\begin{tabular}{lllllllll}
\hline Studie & $N$ & Jahr & Methode & $\begin{array}{l}\text { Hochschul- } \\
\text { Abschluss } \\
\%\end{array}$ & $\begin{array}{l}\text { Pädagogisches } \\
\text { Studium } \\
\%\end{array}$ & $\begin{array}{l}\text { Berufs- } \\
\text { Ausbildung } \\
\%\end{array}$ & $\begin{array}{l}\text { Mindestens } \\
1 \text { ZQ oder } \\
\text { TA } \\
\%\end{array}$ & $\begin{array}{l}\text { TA } \\
\%\end{array}$ \\
\hline A & 31 & 2016 & Interview & 80,6 & 19,4 & 19,4 & 54,8 & 54,8 \\
B & 255 & 2016 & $\begin{array}{l}\text { Online } \\
\text { F }\end{array}$ & 68,8 & 18,3 & 27,5 & 84,5 & 54,9 \\
C & 508 & 2016 & Online & 69,7 & 15,7 & 27,3 & 87,5 & 61,1 \\
D & 15 & 2015 & Interview & 73,3 & 13,3 & 26,7 & 100,0 & 46,7 \\
E & 19 & 2014 & Interview & 73,7 & 5,3 & 26,3 & 100,0 & 31,6 \\
F & 68 & 2012 & Paper-pencil & 82,1 & 18,8 & 17,9 & 92,6 & 61,5 \\
& & & FB & & & & & \\
Gesamt & 896 & - & - & 70,9 & 16,5 & 26,4 & 86,4 & 58,3 \\
\hline
\end{tabular}

Durchführende (mit ggf. Publikation): A: Bonnes und Hochholdinger, B: Wißhak und Hochholdinger (2019), C: Bonnes und Hochholdinger, D: Barth und Hochholdinger (2018), E: Bonnes und Hochholdinger (2016), $F$ : Hochholdinger und Keller (2015a, 2015b)

$Z Q$ Zusatzqualifikation, $T A$ Trainerausbildung, $F B$ Fragebogen

\section{Methode}

\subsection{Datengewinnung}

Die Gesamtstichprobe umfasst 896 Lehrende in der beruflich-betrieblichen Weiterbildung und setzt sich aus den Daten sechs voneinander unabhängiger Studien zusammen, die von den Autorinnen in den vergangenen Jahren durchgeführt wurden (Tab. 3). Bei allen Studien handelt es sich um Gelegenheitsstichproben. Die Befragten wurden über Unternehmen, Weiterbildungsinstitutionen, Internetplattformen, Social Media oder berufliche Netzwerke kontaktiert. Da die Möglichkeit bestand, dass eine Person an mehreren der Studien teilnahm, wurden die Datensätze auf Dopplungen untersucht und die entsprechenden Fälle entfernt. Tab. 3 enthält die Werte der Einzelstudien für die untersuchten Qualifikationen.

Die sechs Studien wurden zusammengeführt, wobei die soziodemografischen und qualifikationsbezogenen Variablen überwiegend identisch waren. In manchen Fällen wurden Antwortkategorien zusammengefasst, oder es wurden unterschiedlich formulierte, semantisch identische Antwortkategorien vereinheitlicht. Für die Überprüfung der Annahmen wurden die Werte aus unserer Stichprobe zu dem gewichteten Mittelwert der Vergleichsstudien in Beziehung gesetzt. Dazu gehören die Untersuchungen von Alfänger et al. (2016), Lenk (2010), Martin und Langemeyer (2014), Marx et al. (2018), Schütz und Nittel (2012) sowie der wb-personalmonitor (Koscheck und Ohly 2016) und die WSF-Studie (2005). Für die Gewichtung des Mittelwerts wurde die Stichprobengröße der einzelnen Vergleichsstudien herangezogen. Da sich die Stichprobe von Fuchs (2011) gänzlich aus Lehrenden in der beruflich-betrieblichen Weiterbildung zusammensetzt, wurde sie nicht für den Vergleich herangezogen. 
Tab. 4 Bildungsabschluss $(N=884)$

\begin{tabular}{lll}
\hline Höchster Bildungsabschluss & $N$ & $\%$ \\
\hline Kein Bildungsabschluss & 22 & 2,5 \\
Berufsausbildung & 233 & 26,4 \\
Hochschulabschluss & 548 & 62,0 \\
Promotion & 79 & 8,9 \\
Sonstige & 2 & 0,2 \\
\hline
\end{tabular}

Rechts-, Wirtschafts- und Sozialwiss.

Päd. bzw. erziehungswiss. Studium Ingenieurwissenschaften

을
Psychologie Geisteswissenschaften Mathematik, Naturwissenschaften Kunst, Kunstwissenschaft Humanmedizin, Gesundheitswiss. Sport
Agrar-, Forst-, Ernährungswiss., Veterinärmedizin

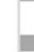

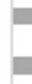
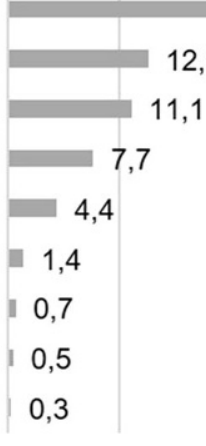

\section{$\begin{array}{llll}0 & 10 & 20 & 30\end{array}$}

Abb. 1 Studienfächer, Anteil der Personen mit Hochschulabschluss in Prozent $(N=586)$, Mehrfachnennungen waren möglich

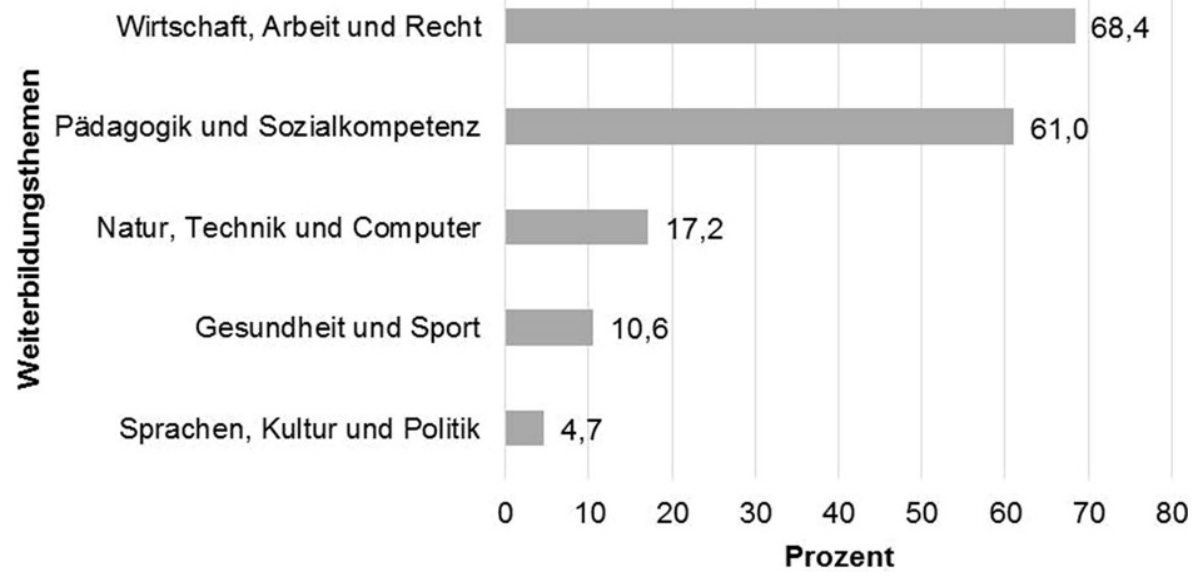

Abb. 2 Angebotene Themen, Anteil der Personen in Prozent $(N=784)$, Mehrfachnennungen waren möglich 


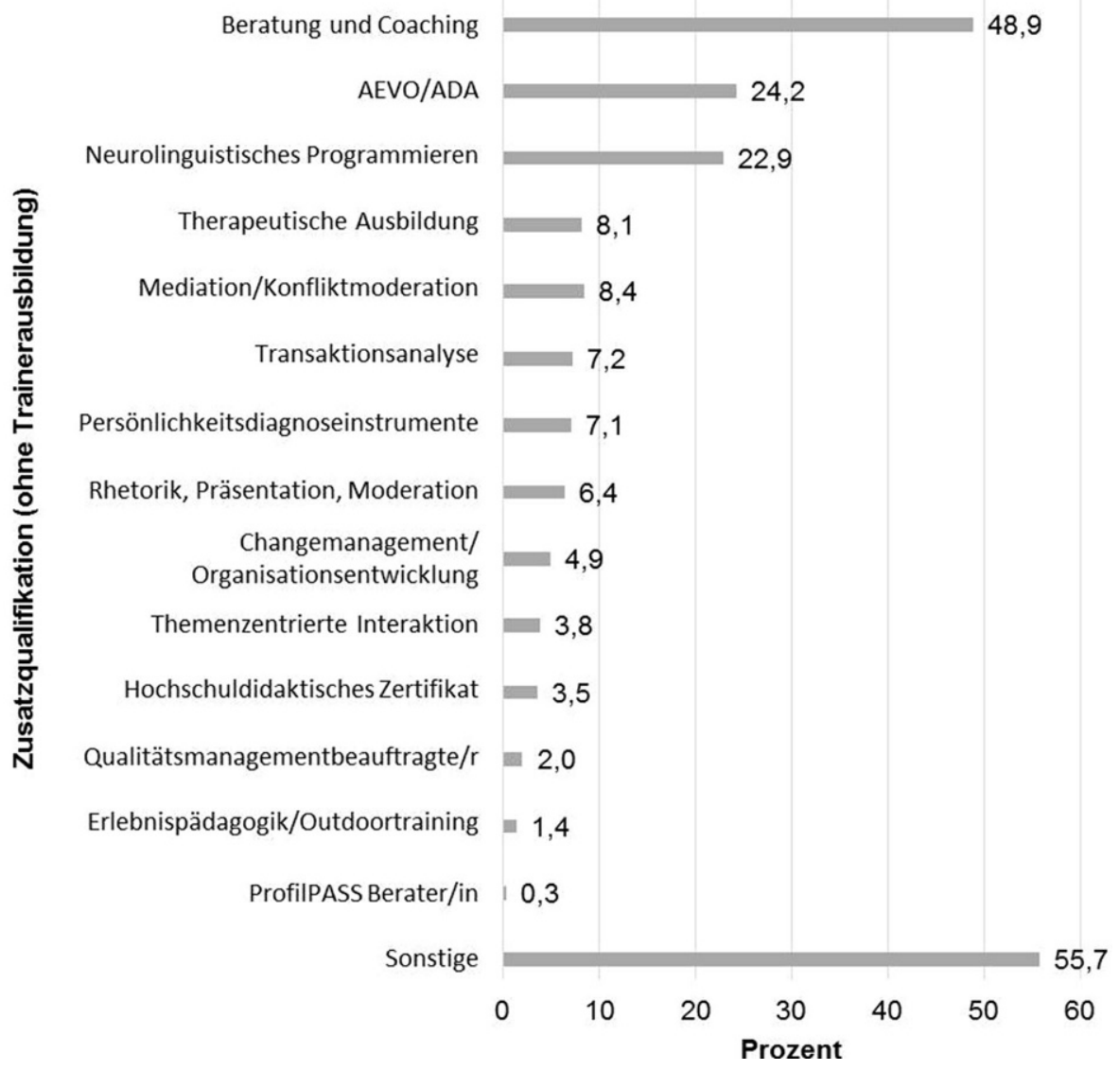

Abb. 3 Zusatzqualifikationen, Anteil der Personen in Prozent $(N=652)$, Mehrfachnennungen waren möglich

\subsection{Stichprobe}

Insgesamt sind 54,7\% Männer und 45,3\% Frauen in der Gesamtstichprobe vertreten $(N=895)$. Das durchschnittliche Alter liegt bei 49,1 Jahren ( $\mathrm{SD}=9,3 ; 22-78$; $N=891)$. Ein hoher Anteil $(70,9 \%)$ der Befragten verfügt über einen Hochschulabschluss (Tab. 4). In Abb. 1 ist zu sehen, wieviel Prozent der Akademiker/innen welche Studienabschlüsse besitzen. Dabei sind die häufigsten Studienfächer die RechtsWirtschafts- und Sozialwissenschaften $(46,1 \%)$, gefolgt von Pädagogik bzw. Erziehungswissenschaften mit $24,7 \%$.

Über die Hälfte (54,5\%) der Befragten üben die Lehrtätigkeit hauptberuflich aus, $23,7 \%$ nebenberuflich und 21,8\% gaben sonstige Erwerbsformen an $(N=803)$. Der Großteil der Teilnehmenden $(81,3 \%)$ gab an, die Lehrtätigkeit ausschließlich im Rahmen einer selbstständigen bzw. freiberuflichen Tätigkeit auszuüben. Nur 10,9\% gaben ausschließlich ein Angestelltenverhältnis an und 5,7\% gaben an, ihre Lehrtätigkeit in beiden Beschäftigungsverhältnissen auszuüben. Ausschließlich ,,sonsti- 
ge Beschäftigungsverhältnisse" gaben $2,1 \%$ an $(N=847)$. Bei der Frage nach den Auftraggebern gaben $78,7 \%$ Unternehmen an $(N=508)$. Die Lehrenden besaßen durchschnittlich 15,5 Jahre Berufserfahrung ( $\mathrm{SD}=8,5 ; 1-50 ; N=873)$ und führten 75 Weiterbildungstage in den letzten 12 Monaten $(\mathrm{SD}=59,7 ; 0-330 ; N=827)$ durch.

Die von den Befragten angebotenen Weiterbildungsthemen waren inhaltlich zu einem großen Teil dem Thema „Wirtschaft, Arbeit und Recht“" zuzuordnen (68,4\%), gefolgt von „Pädagogik und Sozialkompetenz“ mit 61,0\% (Abb. 2).

Ein großer Anteil der Befragten (58,3\%) gab an, eine Trainerausbildung absolviert zu haben $(N=880)$. Darüber hinaus gab es ein großes Spektrum an weiteren Zusatzqualifikationen (Abb. 3). Beratung und Coaching (48,9\%) wurden davon am häufigsten genannt.

\section{Ergebnisse}

Im Folgenden werden die Ergebnisse zu den einzelnen Annahmen dargestellt. Die Referenzwerte der Vergleichsstudien sind in Tab. 2 aufgeführt.

\subsection{Hochschul- und Berufsabschlüsse}

Annahme 1a. Betrachtet man die Fälle, die einen Hochschulabschluss angegeben haben, so liegt der Anteil von 70,9\% $(N=884)$ in unserer Stichprobe ähnlich hoch, wie in den Studien des WSF (2005), von Lenk (2010), Schütz und Nittel (2012), Alfänger et al. (2016), Koscheck und Ohly (2016) und Marx et al. (2018). Nur im Mikrozensus (Martin und Langemeyer 2014) liegt der Anteil mit 45,5\% deutlich niedriger (Abb. 4). Der gewichtete Mittelwert aller Vergleichsstudien liegt bei 65,9\% (gew. $S D=9,4$ ). Damit kann die Annahme, dass der Anteil in beiden Gruppen ähnlich hoch ist, aufrechterhalten werden.

Annahme 1b. Der Anteil der Lehrenden, die ein pädagogisches oder erziehungswissenschaftliches Studium absolviert haben, liegt in unserer Studie bei 16,5\% $(N=879)$. Der Anteil ist vergleichbar mit dem Anteil von 16\% des Mikrozensus (Martin und Langemeyer 2014). In den anderen Studien ist er um ca. ein Drittel (Lenk 2010; Alfänger et al. 2016; Koscheck und Ohly 2016; Marx et al. 2018) oder um ca. das Doppelte (WSF 2005; Schütz und Nittel 2012) höher (Abb. 4). Der gewichtete Mittelwert aller Studien liegt bei 26,6\% (gew. $S D=6,3$ ). Damit lässt sich die Annahme, dass der Anteil eines pädagogischen oder erziehungswissenschaftlichen Studiums ähnlich hoch wie bei Weiterbildungspersonal generell ist, nicht aufrechterhalten.

Annahme 1c. Etwa ein Viertel der Lehrenden in unserer Stichprobe $(26,4 \%$, $N=884$ ) gaben eine Berufsausbildung als den höchsten Abschluss an. Da sich die Anteile der Berufsausbildungen in den Studien zum Weiterbildungspersonal zwischen 10 und $54 \%$ bewegen (WSF 2005; Lenk 2010; Martin und Langemeyer 2014; Alfänger et al. 2016; Koscheck und Ohly 2016) und das gewichtete Mittel bei 29,5 


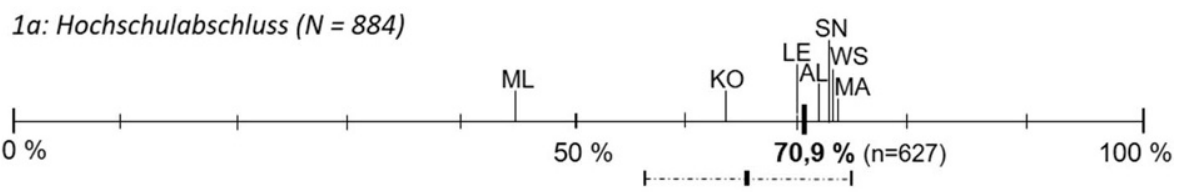

1b: Pädagogisches / erziehungswissenschaftliches Studium ( $N=879)$

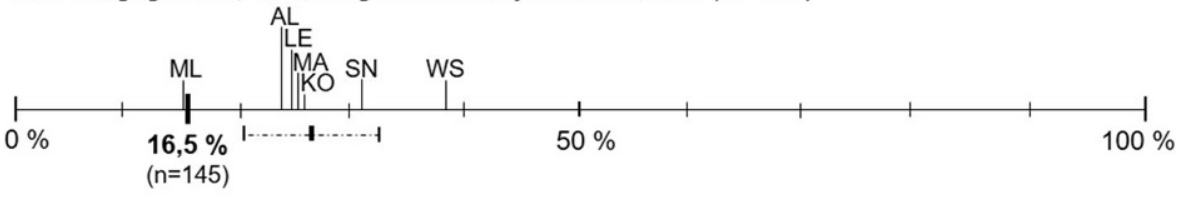

1c: Berufsausbildung $(N=884)$

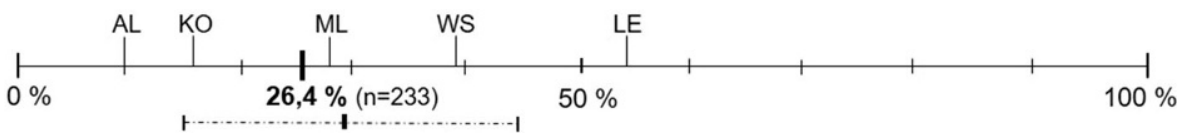

Abb. 4 Gegenüberstellung unserer Befunde und der Ergebnisse der Vergleichsstudien: Studien- und Berufsabschlüsse. ( $A L$ Alfänger et al. (2016), KO Koscheck und Ohly (2016), LE Lenk (2010), MA Marx et al. (2018), ML Martin und Langemeyer (2014), SN Schütz und Nittel (2012), WS WSF (2005). Gestrichelte Linie: gewichteter Mittelwert und gewichtete Standardabweichung der Vergleichsstudien, Balken: Prozentwert der vorliegenden Studie)

(gew. $\mathrm{SD}=15,8$ ) liegt, lässt sich nicht beurteilen, ob hier ein Unterschied vorliegt (Abb. 4).

\subsection{Zusatzqualifikationen}

Annahme 2a. Der Großteil unserer Studienteilnehmenden $(86,4 \%)$ gab an, über eine Trainerausbildung oder eine oder mehrere sonstige Zusatzqualifikationen zu verfügen $(N=891)$. Der Anteil liegt bei Lenk (2010), Alfänger et al. (2016) und Koscheck und Ohly (2016) niedriger (gew. $M=60,7 \%$, gew. $S D=2,4$ ) (Abb. 5), so dass Annahme 2a nicht aufrechterhalten werden kann.

Annahme 2b. Betrachtet man die Häufigkeit von Trainerausbildungen, liegt diese in unserer Stichprobe mit 58,3\% $(N=880)$ deutlich höher als mit $12,6 \%$ bei Koscheck und Ohly (2016) (Abb. 5), so dass die Annahme beibehalten werden kann.

\subsection{Pädagogische Hochschulabschlüsse und Zusatzqualifikationen}

Annahme 3a. Der Großteil der Lehrenden, die einen pädagogischen Hochschulabschluss besitzen, gab auch eine oder mehrere Zusatzqualifikationen an $85,3 \%$, $N=129$ ), während der Anteil mit Zusatzqualifikationen bei den Lehrenden ohne einen pädagogischen Studienabschluss bei 78,4\% liegt $(N=675)$. Der Chi-Qua- 


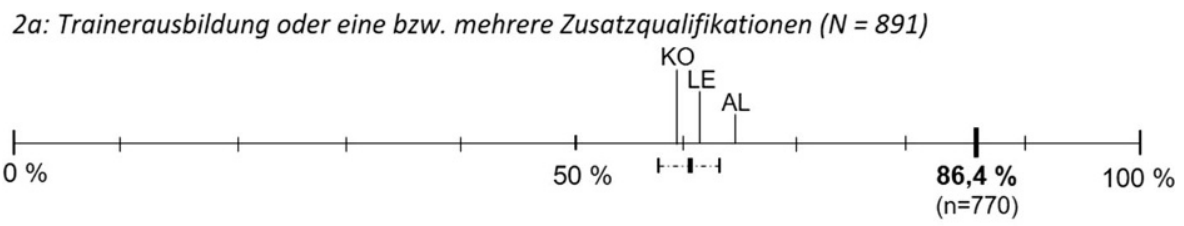

2b: Häufigkeit von Trainerausbildungen $(N=880)$

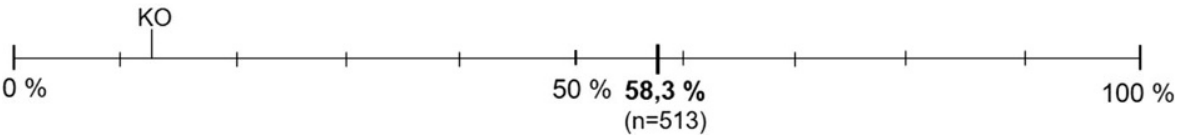

Abb. 5 Gegenüberstellung unserer Befunde und der Ergebnisse der Vergleichsstudien: Zusatzqualifikationen. (AL Alfänger et al. (2016), KO Koscheck und Ohly (2016), LE Lenk (2010). Gestrichelte Linie: gewichteter Mittelwert und gewichtete Standardabweichung der Vergleichsstudien, Balken: Prozentwert der vorliegenden Studie)

drat-Test zeigt keinen signifikanten Unterschied zwischen den beiden Gruppen an $\left(\chi^{2}(1)=3,16, p=0,10\right)$. Damit wird die Annahme beibehalten.

Annahme 3b. Zwei Drittel der Personen (60,1\%, N=143), die einen pädagogischen oder erziehungswissenschaftlichen Hochschulabschluss angaben, haben auch eine Trainerausbildung absolviert, im Vergleich zu 58,0\% der Personen ohne einen solchen Abschluss $(N=724)$. Auch hier erbringt der Chi-Quadrat-Test keinen signifikanten Unterschied $\left(\chi^{2}(1)=0,22, p=0,71\right)$, womit auch hier die Annahme beibehalten wird.

\section{Diskussion}

In der vorliegenden Studie wurden die Qualifikationen von 896 Lehrenden in der beruflich-betrieblichen Weiterbildung beschrieben und mit vorliegenden Befunden zum Weiterbildungspersonal verglichen.

\subsection{Zusammenfassung der Ergebnisse und Einordnung in die aktuelle Befundlage}

Obwohl die Lehrenden in der beruflich-betrieblichen Weiterbildung in unserer Untersuchung, wie in der Weiterbildungsbranche üblich, einen hohen Akademisierungsgrad aufweisen, besitzen sie deutlich seltener pädagogische Hochschulabschlüsse. Dieser Befund deckt sich mit dem Anteil pädagogischer Abschlüsse bei Fuchs (2011, $18 \%$ ). In der Studie von Alfänger et al. (2016), sahen die in der beruflich-betrieblichen Weiterbildung Beschäftigten eine pädagogische Qualifikation am seltensten als Voraussetzung für ihre Position an ( $37 \%$ gegenüber $52 \%$ bei den anderen Gruppen). Dies ist ggf. damit zu erklären, dass Fachspezialist/innen aufgrund ihrer jeweiligen Expertise (bspw. im Vertrieb oder bezüglich einer bestimmten Software) zum Teil erst im Laufe ihrer Berufsbiografie eine Lehrtätigkeit in der beruflich-betrieblichen 
Weiterbildung aufnehmen (Bonnes und Hochholdinger 2016; Fuchs 2011). In dem Fall haben sie oft eine Ausbildung oder ein Fachstudium absolviert, besitzen jedoch keine formale pädagogische Qualifikation, welche von Auftrag- oder Arbeitgebern ggf. auch nicht erwartet wird. Für diese Erklärung sprechen auch die Befunde von Koscheck (2018), denen zufolge Weiterbildungsanbieter auf dem privaten Markt bei der Personalrekrutierung vor allem auf einschlägige fachliche Berufserfahrung achten und weniger auf formale pädagogische Qualifikationen. Entsprechend besitzen Hochrechnungen des wb-personalmonitors und des wbmonitors zufolge auch nur $15,3 \%$ der Lehrenden auf dem privaten Markt ein pädagogisches Studium (Koscheck 2018).

Unabhängig davon, ob sie pädagogisch vorgebildet sind oder nicht, scheinen Lehrende in der beruflich-betrieblichen Weiterbildung häufiger verschiedene Zusatzqualifikationen zu besitzen. Da sich die Lehrenden in der beruflich-betrieblichen Weiterbildung oft als Trainer/innen verstehen, ist es plausibel, dass Trainerausbildungen die häufigste Zusatzqualifikation darstellen (Fuchs 2011). Auch bei Koscheck (2018) besaßen die Lehrenden, die für Anbieter am privaten Weiterbildungsmarkt tätig waren, überdurchschnittlich häufig eine Trainerausbildung.

Dass viele Befragte auch eine Zusatzausbildung in Beratung und/oder Coaching besitzen (48,9\%), entspricht den Ergebnissen von Fuchs (2011) zu Tätigkeiten von Trainer/innen, denen zufolge Beratung und Coaching zusammen etwa $21 \%$ ihrer Arbeitszeit ausmachen. Auch in der Erhebung von Alfänger et al. (2016) gaben die Beschäftigten in der beruflich-betrieblichen Weiterbildung häufiger Zusatzqualifikationen im Bereich Beratung, Coaching oder Mediation an. Eine mögliche Erklärung, für die uns jedoch keine empirische Evidenz vorliegt, ist, dass Trainer/innen teilweise auch als Coaches arbeiten.

\subsection{Implikationen für die Professionalisierung der Lehrenden in der beruflich- betrieblichen Weiterbildung}

Der vorliegende Beitrag beschäftigt sich mit einer Teilgruppe des Weiterbildungspersonals, die bisher in der erwachsenenpädagogischen Professionalisierungsforschung wenig explizite Berücksichtigung fand. Die Vergleiche der Qualifikationen von Lehrenden in der beruflich-betrieblichen Weiterbildung mit den bisherigen Befunden zum Weiterbildungspersonal ergaben, dass, neben grundlegenden Gemeinsamkeiten wie dem hohen Qualifikationsniveau, auch einige Besonderheiten bestehen. So befinden sich unter den Lehrenden in der beruflich-betrieblichen Weiterbildung weniger genuin pädagogisch ausgebildete Personen. Dies sollte bei Professionalisierungsbestrebungen mitbedacht werden. Vor dem Hintergrund, dass erziehungswissenschaftliche Studiengänge eher auf eine leitende bzw. planende Tätigkeit in der Weiterbildung vorbereiten als auf eine Lehrtätigkeit (Wißhak und Hochholdinger 2016), lässt sich aus dem Fehlen eines solchen Abschlusses jedoch nicht zwangsläufig auf mangelnde Lehrprofessionalität schließen. Marx et al. (2018) fanden bspw. keine Zusammenhänge zwischen einem pädagogischen Studienabschluss und dem Wissen über Lehr-Lernmethoden und -konzepte bei Lehrenden aus der Weiterbildung.

Lehrende in der beruflich-betrieblichen Weiterbildung scheinen einen starken Fokus auf die eigene Weiterbildung zu legen. Es wäre noch zu prüfen, inwiefern diese 
heterogenen Zusatzqualifikationen tatsächlich zu einer Erhöhung der professionellen Handlungskompetenz beitragen. In unserer Stichprobe stehen die Trainerausbildungen an erster Stelle. Eine Analyse dieser Angebote (Wißhak und Hochholdinger 2015) weist darauf hin, dass sie inhaltlich und qualitativ recht divers sind und viel methodisches Rüstzeug, jedoch wenig evidenzbasiertes bzw. wissenschaftlich fundiertes Wissen vermitteln.

Für die Professionalisierung der Lehrenden in der Weiterbildung dürfte in $\mathrm{Zu}$ kunft auch das Projekt GRETA eine Rolle spielen. Auf der Basis eines eigens entwickelten Kompetenzmodells ermöglicht es einerseits die Validierung non-formal und informell erworbener Kompetenzen der Lehrenden wie auch den Abgleich mit Curricula von Train-the-Trainer Ausbildungen (Bosche et al. 2019). Aktuell werden die verschiedenen Nutzungsmöglichkeiten im Folgeprojekt GRETA II pilotiert.

\subsection{Limitationen und Forschungsdesiderata}

Bei den sechs zusammengefassten Einzelstudien handelt es sich um Gelegenheitsstichproben, so dass die Ergebnisse nicht als repräsentativ zu verstehen sind. Die Gelegenheitsstichproben sind in voneinander unabhängigen Projektkontexten entstanden, jedoch besteht die Möglichkeit eines selektiven Effektes für die Gesamtstichprobe. Darüber hinaus legen Studie B und C die Datengrundlage für ca. $85 \%$ der Gesamtstichprobe (Tab. 3). Aufgrund der Datenherkunft aus verschiedenen Erhebungen liegen den Auswertungen einzelner Variablen z.T. verschiedene Fallzahlen zugrunde. Dennoch handelt es sich nach unserem Wissen um die größte Stichprobe von Lehrenden in der beruflich-betrieblichen Weiterbildung in Deutschland, die zur Untersuchung ihrer Qualifikationen herangezogen wurde. Aufgrund der nicht immer genau ermittelbaren Fallzahl $(n)$, die den Prozentangaben in den Vergleichsstudien zugrunde liegt, konnten die Unterschiede zwischen unserer Gesamtstichprobe und den Vergleichsstudien nicht auf Signifikanz überprüft werden. Die Übersicht über die einzelnen Werte für alle Vergleichsstudien (Tab. 2) ermöglicht es jedoch, unsere Einschätzung der Unterschiede transparent zu machen.

Für zukünftige Erhebungen wäre es jedoch wünschenswert, eine repräsentative Stichprobe zu befragen. Dadurch ließe sich die Vermutung, dass Lehrende in der beruflich-betrieblichen Weiterbildung spezifische Qualifikationsmerkmale aufweisen, fundierter beurteilen.

Während das Ziel der vorliegenden Studie ist, die Qualifikationen von Lehrenden in der beruflich-betrieblichen Weiterbildung quantitativ zu beschreiben, können keine Aussagen über die Qualität und Passung der verschiedenen Qualifikationen abgeleitet werden. So wäre bspw. zu erwarten, dass manche Zusatzqualifikationen, wie Train-the-Trainer-Zertifikate, besser auf eine Lehrtätigkeit in der Weiterbildung vorbereiten als bspw. die Ausbildereignung nach AEVO, die stärker auf die berufliche Ausbildung abzielt. Während für Trainerausbildungen und erziehungswissenschaftliche Studiengänge erste Curriculumanalysen vorliegen (Wißhak und Hochholdinger 2015, 2016), müsste auch für weitere Angebote untersucht werden, inwiefern sie für eine Lehrtätigkeit in der Weiterbildung nützlich sind.

Da die Qualifikationen, seien es nun Hochschulabschlüsse oder Zusatzqualifikationen, noch keine Schlussfolgerungen darüber zulassen, welche professionellen 
Kompetenzen tatsächlich erworben wurden, ist außerdem die Erfassung dieser Kompetenzen wünschenswert. Der von Marx et al. (2014, 2017) entwickelte Test zur Erfassung des bildungsbereichsübergreifenden pädagogisch-psychologischen Wissens von Lehrkräften ist ein erster Schritt in diese Richtung.

Zusätzlich müsste man die von den Lehrenden angebotenen Weiterbildungsthemen und -inhalte in zukünftigen Studien mitberücksichtigen. So wäre zu untersuchen, ob je nach Weiterbildungsthema - abgesehen von den unterschiedlichen Anforderungen an die fachliche Qualifikation - auch unterschiedliche didaktische Anforderungen an die Lehrenden bestehen. Laker und Powell (2011) gehen davon aus, dass verhaltensorientierte Softskill-Themen wie Kommunikation oder Selbstmanagement anders gelehrt werden müssen als wissensorientierte, technische Themen. Somit wären gegebenenfalls unterschiedliche Kompetenzen der Lehrenden vonnöten.

Während bezüglich der Kompetenz- und Professionalitätsentwicklung der Lehrenden weiterhin ein großer konzeptioneller und forscherischer Bedarf besteht, liefert dieser Beitrag zunächst einen quantitativen, deskriptiven Überblick über die Qualifikationen. Daraus lässt sich ableiten, dass Lehrende in der beruflich-betrieblichen Weiterbildung bezüglich ihrer Qualifikationen einige Spezifika aufweisen und bei Professionalisierungsbestrebungen als Subgruppe des Weiterbildungspersonals berücksichtigt werden sollten.

Funding Open Access funding provided by Projekt DEAL.

Open Access Dieser Artikel wird unter der Creative Commons Namensnennung 4.0 International Lizenz veröffentlicht, welche die Nutzung, Vervielfältigung, Bearbeitung, Verbreitung und Wiedergabe in jeglichem Medium und Format erlaubt, sofern Sie den/die ursprünglichen Autor(en) und die Quelle ordnungsgemäß nennen, einen Link zur Creative Commons Lizenz beifügen und angeben, ob Änderungen vorgenommen wurden.

Die in diesem Artikel enthaltenen Bilder und sonstiges Drittmaterial unterliegen ebenfalls der genannten Creative Commons Lizenz, sofern sich aus der Abbildungslegende nichts anderes ergibt. Sofern das betreffende Material nicht unter der genannten Creative Commons Lizenz steht und die betreffende Handlung nicht nach gesetzlichen Vorschriften erlaubt ist, ist für die oben aufgeführten Weiterverwendungen des Materials die Einwilligung des jeweiligen Rechteinhabers einzuholen.

Weitere Details zur Lizenz entnehmen Sie bitte der Lizenzinformation auf http://creativecommons.org/ licenses/by/4.0/deed.de.

\section{Literatur}

Alfänger, J., Cywinski, R., \& Elias, A. (2016). Beschäftigung in der Weiterbildung. Der gespaltene Weiterbildungsmarkt im Spanungsfeld von Profession und Prekarität. Dissertation, Universität DuisburgEssen: Essen.

Autorengruppe wb-personalmonitor (2016). Das Personal in der Weiterbildung. Arbeits- und Beschäftigungsbedingungen, Qualifikationen, Einstellungen zu Arbeit und Beruf. DIE Survey. Bielefeld: wbv.

Barth, D., \& Hochholdinger, S. (2018). Wie unterstützen Lehrende den Trainingstransfer in Führungstrainings? Eine qualitative Interviewstudie. Bildungsforschung, 1, 1-18. URN:urn:nbn:de:0111-pedocs165773.

Bilger, F., \& Strauß, A. (2017). Beteiligung an non-formaler Weiterbildung. In F. Bilger, F. Behringer, H. Kuper \& J. Schrader (Hrsg.), Bildung in Deutschland 2016. Ein indikatorengestützter Bericht mit einer Analyse zu Bildung und Migration (S. 25-55). Bielefeld: wbv.

Bonnes, C., \& Hochholdinger, S. (2016). Beruf TrainerIn? - Die berufliche Entwicklung und die Wege des Qualifikations- und Kompetenzerwerbs des Weiterbildungspersonals in der beruflichen und betrieb- 
lichen Weiterbildung. bwp@,(29 Update). http://www.bwpat.de/ausgabe29/bonnes_hochholdinger_ bwpat29.pdf. Zugegriffen: 31.05.2016

Bosche, B., Strauch, A. \& Schneider, M., Brandt, P. (2019). GRETA-Anerkennungsverfahren: adaptiv statt one-fits all. Kompetenzvalidierung von Lehrenden zwischen diversen Ansprüchen und Nutzenvorstellungen. Magazin erwachsenenbildung.at., 37(2). https://erwachsenenbildung.at/magazin/19-37/ meb19-37.pdf. Zugegriffen: 31.10.2019

Diez, M.E. (2010). It is complicated. Unpacking the flow of teacher education's impact on student learning. Journal of Teacher Education, 61(5), 441-450.

Dobischat, R., Elias, A., \& Rosendahl, A. (Hrsg.). (2018). Das Personal in der Weiterbildung. Im Spannungsfeld von Professionsanspruch und Beschäftigungsrealität. Wiesbaden: VS.

Fuchs, S. (2011). Professionalitätsentwicklung des Weiterbildungspersonals. Tätigkeiten, Kompetenzen und Fortbildung von Trainern in der beruflichen/betrieblichen Weiterbildung. Hamburg: Dr. Kovac.

Gieseke, W. (2018). Professionalität und Professionalisierung in der Erwachsenenbildung/Weiterbildung. In R. Tippelt \& A. von Hippel (Hrsg.), Handbuch Erwachsenenbildung/Weiterbildung (Bd. 1, S. 1051-1069). Wiesbaden: Springer. https://doi.org/10.1007/978-3-531-19979-5_52.

Hochholdinger, S., \& Keller, I. (2015a). Welche Zusammenhänge bestehen zwischen den affektiven Reaktionen von Trainingsteilnehmenden, der Lehrorientierung von Weiterbildungspersonal und Weiterbildungsinhalten? Zeitschrift für Erziehungswissenschaft, 18(S1), 123-144. https://doi.org/10.1007/ s11618-014-0602-6.

Hochholdinger, S., \& Keller, I. (2015b). Emotionales Erleben in betrieblichen Weiterbildungen. In A. Rausch, J. Warwas, J. Seifried \& E. Wuttke (Hrsg.), Konzepte und Ergebnisse ausgewählter Forschungsfelder der beruflichen Bildung. Festschrift für Detlef Sembill (1. Aufl. S. 253-269). Baltmannsweiler: Schneider Hohengehren.

Käpplinger, B., \& Lichte, N. (2012). Erhöhung der Weiterbildungsbeteiligung durch professionelles Weiterbildungspersonal. WSI Mitteilungen, 5, 374-381.

Koscheck, S. (2018). Pädagogische Professionalität in Teilsegmenten der Weiterbildung. In R. Dobischat, A. Elias \& A. Rosendahl (Hrsg.), Das Personal in der Weiterbildung. Im Spannungsfeld von Professionsanspruch und Beschäftigungsrealität (S. 161-183). Wiesbaden: Springer.

Koscheck, S., \& Martin, A. (2016). Standardisierte Befragungen des Personals in der Erwachsenen- und Weiterbildung. In Autorengruppe wb-personalmonitor (Hrsg.), Das Personal in der Weiterbildung. Arbeits- und Beschäftigungsbedingungen, Qualifikationen, Einstellungen zu Arbeit und Beruf. DIE Survey. (S. 30-51). Bielefeld: wbv.

Koscheck, S., \& Ohly, H. (2016). Qualifikationen des Personals in der Erwachsenen- und Weiterbildung. In Autorengruppe wb-personalmonitor (Hrsg.), Das Personal in der Weiterbildung. Arbeitsund Beschäftigungsbedingungen, Qualifikationen, Einstellungen zu Arbeit und Beruf. DIE Survey. (S. 107-131). Bielefeld: wbv.

Kunina-Habenicht, O., Schulze-Stocker, F., Kunter, M., Baumert, J., Leutner, D., Förster, D., Lohse-Bossenz, H., \& Terhart, E. (2013). Die Bedeutung der Lerngelegenheiten im Lehramtsstudium und deren individuelle Nutzung für den Aufbau des bildungswissenschaftlichen Wissens. Zeitschrift für Pädagogik, 59(1), 1-23.

Kunter, M., Baumert, J., Blum, W., Klusmann, U., Krauss, S., \& Neubrand, M. (Hrsg.). (2013). Cognitive activation in the mathematics classroom and professional competence of teachers. Results from the COACTIV project. Mathematics teacher education, Bd. 8. New York, Heidelberg, Dortrecht, London: Springer.

Laker, D. R., \& Powell, J.L. (2011). The differences between hard and soft skills and their relative impact on training transfer. Human Resource Development Quarterly, 22(1), 111-122. https://doi.org/10. 1002/hrdq. 20063 .

Lenk, C. (2010). Freiberufler in der Weiterbildung. Empirische Studie am Beispiel Hessen. Erwachsenenbildung und lebensbegleitendes Lernen, Bd. 16. Bielefeld: Bertelsmann.

Martin, A. (2016). Tätigkeiten des Weiterbildungspersonals. In Autorengruppe wb-personalmonitor (Hrsg.), Das Personal in der Weiterbildung. Arbeits- und Beschäftigungsbedingungen, Qualifikationen, Einstellungen zu Arbeit und Beruf. DIE Survey. (S. 97-106). Bielefeld: wbv.

Martin, A., \& Langemeyer, I. (2014). Demografie, sozioökonomischer Status und Stand der Professionalisierung - das Personal in der Weiterbildung im Vergleich. In Deutsches Institut für Erwachsenenbildung (Hrsg.), Trends der Weiterbildung. DIE-Trendanalyse 2014. DIE spezial. (S. 43-67). Bielefeld: Bertelsmann.

Martin, A., Lencer, S., \& Schrader, J. (2016). Das Personal in der Erwachsenen- und Weiterbildung. In Autorengruppe wb-personalmonitor (Hrsg.), Das Personal in der Weiterbildung. Arbeits- und Beschäf- 
tigungsbedingungen, Qualifikationen, Einstellungen zu Arbeit und Beruf. DIE Survey. (S. 11-25). Bielefeld: wbv.

Marx, C., Goeze, A., Kelava, A., \& Schrader, J. (2018). Lehrkräfte in der Erwachsenen- und Weiterbildung - Zusammenhänge zwischen Vorbildung und Erfahrung mit dem Wissen über Lehr-Lernmethoden und -konzepte. Zeitschrift für Weiterbildungsforschung - Report, 41(1), 57-77. https://doi.org/10. 1007/s40955-018-0108-6.

Marx, C., Goeze, A., \& Schrader, J. (2014). Adult education teachers' pedagogical-psychological knowledge. Potential elements and test development. In W. Jütte \& S. Lattke (Hrsg.), International and comparative perspectives in the field of professionalization (S. 165-182). Frankfurt a.M.: Peter Lang.

Marx, C., Goeze, A., Voss, T., Hoehne, V., Klotz, V. K., \& Schrader, J. (2017). Pädagogisch-psychologisches Wissen von Lehrkräften aus Schule und Erwachsenenbildung: Entwicklung und Erprobung eines Testinstruments. Zeitschrift Für Erziehungswissenschaft, 20(Suppl 1), 165-200. https://doi.org/ 10.1007/s11618-017-0733-7.

Schütz, J., \& Nittel, D. (2012). Von der Heterogenität zur Vielfalt. Akademische Professionalisierung im Blick einer komparativen pädagogischen Berufsgruppenforschung. In R. Egetenmeyer \& D. Nittel (Hrsg.), Akademische Professionalisierung in der Erwachsenenbildung/Weiterbildung. Grundlagen der Berufs- und Erwachsenenbildung, (Bd. 70, S. 229-244). Baltmannsweiler: Schneider Hohengehren.

Wißhak, S., \& Hochholdinger, S. (2015). „Zaubern“ lernen - Welche pädagogischen Inhalte umfassen sogenannte Trainerausbildungen? Zeitschrift für Weiterbildungsforschung - Report, 38(1), 113-127. https://doi.org/10.1007/s40955-015-0012-2.

Wißhak, S., \& Hochholdinger, S. (2016). Analyse der Inhalte erziehungswissenschaftlicher Studiengänge im Hinblick auf eine spätere Tätigkeit in der berufsbezogenen Weiterbildung. Zeitschrift für Weiterbildungsforschung - Report, 39(1), 97-115. https://doi.org/10.1007/s40955-016-0054-0.

Wißhak, S., \& Hochholdinger, S. (2019). Trainers' knowledge and skills from the perspective of trainers, trainees and human resource development practitioners. International Journal of Training Research, 16(3), 218-231. https://doi.org/10.1080/14480220.2018.1576327.

WSF Wirtschafts- und Sozialforschung (2005). Erhebung zur berufichen und sozialen Lage von Lehrenden in Weiterbildungseinrichtungen. Schlussbericht. Kerpen: BMBF. 